\title{
Immune activation and response to pembrolizumab in POLE-mutant endometrial cancer
}

\author{
Janice M. Mehnert, ${ }^{1,2,3}$ Anshuman Panda, ${ }^{1,4}$ Hua Zhong, ${ }^{1}$ Kim Hirshfield, ${ }^{1,3}$ Sherri Damare, ${ }^{1,2}$ Katherine Lane, ${ }^{1}$ Levi Sokol, ${ }^{5}$ \\ Mark N. Stein,, ${ }^{1,2,3}$ Lorna Rodriguez-Rodriquez, ${ }^{1,6}$ Howard L. Kaufman, ${ }^{1,2,7}$ Siraj Ali, ${ }^{8}$ Jeffrey S. Ross, ${ }^{8}$ Dean C. Pavlick, ${ }^{8}$ \\ Gyan Bhanot, ${ }^{1,4,9}$ Eileen P. White, ${ }^{1,9}$ Robert S. DiPaola, ${ }^{1,3}$ Ann Lovell, ${ }^{10}$ Jonathan Cheng, ${ }^{10}$ and Shridar Ganesan ${ }^{1,3}$ \\ ${ }^{1}$ Rutgers Cancer Institute of New Jersey (CINJ), New Brunswick, New Jersey, USA. ²Developmental Therapeutics/Phase I Program, Rutgers CINJ, New Brunswick, New Jersey, USA \\ ${ }^{3}$ Department of Medicine, Rutgers Robert Wood Johnson Medical School, Piscataway, New Jersey USA. ${ }^{4}$ Department of Physics, Rutgers University, Piscataway, New Jersey, USA. ${ }^{5}$ Department of Radiology, \\ ${ }^{6}$ Department of Obstetrics and Gynecology, and ${ }^{7}$ Department of Surgery, Rutgers Robert Wood Johnson Medical School, Piscataway, New Jersey, USA. ${ }^{8}$ Foundation Medicine, Cambridge, Massachusetts, USA. \\ ${ }^{9}$ Molecular Biology and Biochemistry, Rutgers University, Piscataway, New Jersey, USA. ${ }^{10}$ Merck \& Co., Kenilworth, New Jersey, USA.
}

\begin{abstract}
Antibodies that target the immune checkpoint receptor programmed cell death protein 1 (PD-1) have resulted in prolonged and beneficial responses toward a variety of human cancers. However, anti-PD-1 therapy in some patients provides no benefit and/or results in adverse side effects. The factors that determine whether patients will be drug sensitive or resistant are not fully understood; therefore, genomic assessment of exceptional responders can provide important insight into patient response. Here, we identified a patient with endometrial cancer who had an exceptional response to the anti-PD-1 antibody pembrolizumab. Clinical grade targeted genomic profiling of a pretreatment tumor sample from this individual identified a mutation in DNA polymerase epsilon (POLE) that associated with an ultramutator phenotype. Analysis of The Cancer Genome Atlas (TCCA) revealed that the presence of POLE mutation associates with high mutational burden and elevated expression of several immune checkpoint genes. Together, these data suggest that cancers harboring POLE mutations are good candidates for immune checkpoint inhibitor therapy.
\end{abstract}

\section{Introduction}

Prolonged and deep responses to antibody therapy directed against the immune checkpoint programmed cell death protein 1 (PD-1) receptor have been demonstrated in multiple types of human cancer (1-7). However, objective responses are seen in less than half of patients treated, and the potential for serious side effects exists. Combination immune checkpoint inhibitor therapy raises response rates, but also increases toxicity and cost $(8,9)$. There is a clear need for identifying biomarkers of response to immune checkpoint inhibitor therapy, particularly if these markers can select the subset of patients who will require only single-agent anti-PD-1 therapy.

High expression of the programmed cell death ligand 1 (PDL1) is under investigation as a potential predictor of response to anti-PD-1 therapy. However, while patients with higher PD-L1

Conflict of interest: J.M. Mehnert is the principal investigator for clinical trial MK3475-028/KEYNOTE-28, funded by Merck \& Co. (ClinicalTrials.gov NCT02054806). H.L. Kaufman serves on a scientific advisory board and a speaker's bureau for Merck \& Co. (funds returned to Rutgers University); serves on scientific advisory boards for Alkermes, Amgen, Bristol-Myers Squibb, EMD Serono Inc., and Prometheus Laboratories Inc.; provides consulting services for Sanofi; and receives clinical trial funding from Amgen, EMD Serono Inc., Prometheus Laboratories Inc., and Viralytics. S. Ali, J.S. Ross, and D.C. Pavlik are employees of Foundation Medicine. A. Lovell and J. Cheng are employees of Merck \& Co. S. Ganesan serves on a scientific advisory board and as a consultant to Inspirata and has equity in Inspirata; serves on an advisory board for Novartis Pharmaceuticals Corp.; and has a patent (PCT/US2010/022891) on digital imaging technology licensed to Inspirata.

Submitted: October 8, 2015; Accepted: March 15, 2016.

Reference information: J Clin Invest. 2016;126(6):2334-2340. doi:10.1172/JCI84940. expression may experience greater benefit, a subset of patients with PD-L1-negative cancers sustain important responses $(5,8$, 10). Thus, the discovery of additional predictive markers may complement the utility of assessment of PD-L1 expression.

Several emerging studies demonstrate that tumors with high mutational burdens exhibit a greater response rate to immune checkpoint blockade (11-14). Here, we report the genomic analysis of what we believe is the first known case of a patient with endometrial cancer who experienced a prolonged response to pembrolizumab therapy in a clinical trial. Clinical Laboratory Improvement Amendments-certified (CLIA-certified) targeted genomic profiling of a pretreatment biopsy specimen revealed that this tumor had a mutation in the DNA polymerase epsilon (POLE) gene. This $P O L E$ mutation is associated with disruption of the exonuclease activity required for proofreading function and results in a high mutational burden or "ultramutator" phenotype $(15,16)$. POLE mutations are seen in approximately $10 \%$ of endometrial cancers and are associated with increased expression of PD-1 and PD-L1, additional T cell markers (16-20), and robust lymphocytic infiltration. These data suggest that presence of $P O L E$ mutations may identify a subset of cancers especially vulnerable to immune checkpoint therapy.

\section{Results and Discussion}

A 53-year-old woman presenting with irregular vaginal bleeding was treated with hysterectomy and diagnosed with a p $1 \mathrm{~b}$ pNO, stage IB, FIGO grade III endometrial adenocarcinoma, high-grade endometrioid type, with extensive necrosis, lymphovascular invasion, and myometrial invasion. A peritumoral lym- 
A

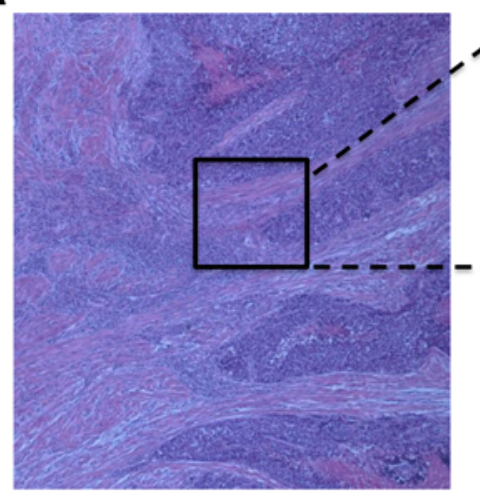

D

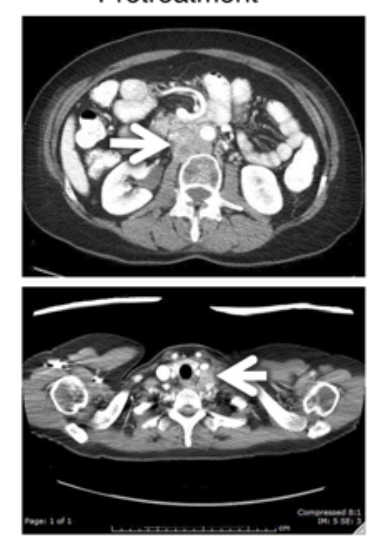

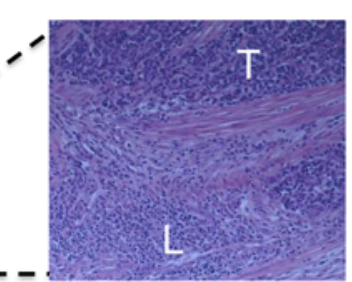

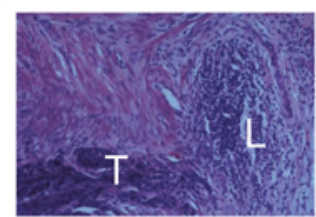

8 weeks pembrolizumab

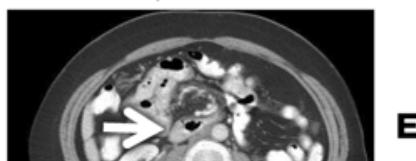

B

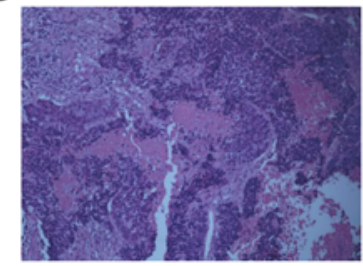

C

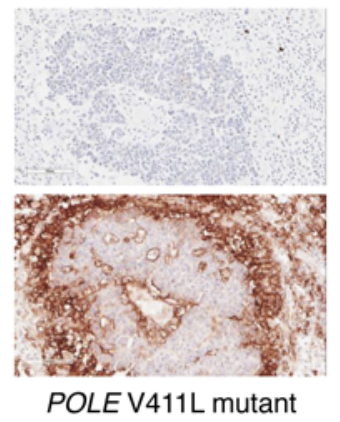

Primary

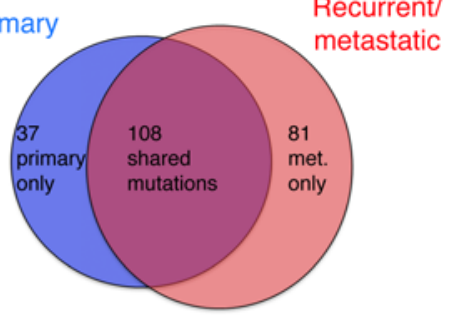

Figure 1. Histologic, radiologic, and genomic characteristics of a patient with POLE-mutant endometrial cancer responding to pembrolizumab. (A) Histology from surgical resection of primary endometrial cancer. Top inset shows region of peritumoral lymphocytic infiltration; bottom inset shows separate region with peritumoral lymphocytic micronodules. Original magnification, $\times 10$ (left); $\times 20$ (right). T, tumor; L, lymphocytic infiltrate. (B) Histology from supraclavicular LN biopsy taken 4 years after original diagnosis. Original magnification, $\times 20$. (C) Sections of LN metastasis: hematoxylin counterstain (negative control; top image); IHC staining with anti-PD-L1 antibody clone 22C3 (Merck) (bottom image). Original magnification, $\times 20$. (D) Representative abdominal (top) and thoracic (bottom) CT images taken prior to pembrolizumab treatment and 8 weeks after initiation of therapy. Arrows highlight paraaortic and supraclavicular tumor masses, which substantially decreased. (E) Number of nonsynonymous somatic variants, including somatic variants of unknown significance, found in 315 cancer-related genes shown for primary and recurrent tumor samples.

phocytic infiltrate was readily apparent (Figure 1A) in her tumor. Her oncologic family history included 2 brothers with prostate cancer, aged 55 and 67 years at diagnosis, a father with a form of brain cancer who died from this disease at age 54 years, and a maternal aunt who was diagnosed with colon cancer at age 54 years and lymphoma, type unknown, a few years later. The patient deferred radiation therapy and, in follow-up, rapidly developed new retroperitoneal adenopathy, with biopsy showing recurrent cancer. She was treated with doxorubicin, cisplatin, paclitaxel, and extended-field radiotherapy. Two years later, she developed supraclavicular adenopathy that, when biopsied, revealed recurrent metastatic adenocarcinoma (Figure 1B). In the process of performing screening tests to determine the eligibility of the patient for a clinical trial of pembrolizumab, this metastasis was found to be positive for PD-L1 expression (Figure 1C) using a prototype immunohistochemical (IHC) assay as previously described (21). CT scans showed bulky retroperitoneal and abdominal lymphadenopathy encasing the inferior vena cava (Figure 1D). She soon developed extensive bilateral lower extremity edema, interfering with her daily activities and quality of life.
The patient enrolled in a phase I trial of pembrolizumab (10 $\mathrm{mg} / \mathrm{kg}$ every 2 weeks) for patients with tumors harboring PD-L1 expression. She experienced rapid clinical improvement, with resolution of lower extremity edema, supraclavicular adenopathy, and a partial response at 8 weeks following treatment initiation, sustained, at this writing, for over 14 months (Figure 1D). She tolerated therapy well, with grade 1 rash, grade 1 liver function test elevation, and grade 2 fever early in her treatment course that resolved spontaneously. She provided informed consent to participate in the Rutgers CINJ genomic tumor-profiling protocol.

The primary endometrial cancer specimen and the recurrent supraclavicular lymph node (LN) metastasis obtained prior to treatment were sent for hybrid-capture-based comprehensive genomic profiling, targeting all exons of 315 cancer-related genes at a CLIA-certified laboratory (Foundation Medicine). There were 32 sequence variants identified as likely pathogenic in the primary tumor and 33 potentially pathogenic variants identified in the LN recurrence; 28 changes were present in both samples (Table 1). Both samples harbored a mutation in the exonuclease domain of POLE that affects proofreading function $(\mathrm{V} 411 \mathrm{~L})$ as well as a separate nonsense mutation in 


\section{Table 1. Likely pathogenic variants identified by targeted sequencing ${ }^{A}$}

\begin{tabular}{|c|c|}
\hline Primary cancer & Metastatic recurrence ( 4 years later) \\
\hline POLE V411L, POLE R114* & POLE V411L, POLE R114*B \\
\hline ERBB3 V104L & ERBB3 V104L \\
\hline TP53 R213* & TP53 R213* \\
\hline MAP2K1 D67N & MAP2K1 D67N \\
\hline РІКЗСА К111N & РІКЗСА К111N \\
\hline PIK3CA R357Q & PIK3CA R357Q \\
\hline PIK3СA R88Q & PIK3CA R88Q \\
\hline PTEN R130Q & PTEN R130Q \\
\hline ATR R989C & ATR R989C \\
\hline CTNNB1 D32G & CTNNB1 D32C \\
\hline XP01 R749W & XP01 R749W \\
\hline ARID1A E1767* & ARID1A E1767* \\
\hline ARID1A R1989* & ARID1A R1989* \\
\hline ATRX E1298* & ATRX E1298* \\
\hline ATRX E585* & ATRX E585* \\
\hline BAP1A301T & BAP1A301T \\
\hline EZH2 E246* & EZH2 E246* \\
\hline FAM123B R225I & FAM123B R225I \\
\hline FAT1 E3190* & FAT1 E3190* \\
\hline FAT1 E840* & FAT1 E840* \\
\hline LRP1B E428* & LRP1B E428* \\
\hline LRBP1B E4584* & LRBP1B E4584* \\
\hline LRBP1B N210H & LRBP1B N210H \\
\hline MSH6 R922Q & MSH6 R922Q \\
\hline NOTCH2 E497* & NOTCH2 E497* \\
\hline SMAD2 R321Q & SMAD2 R321Q \\
\hline STAG2 E1209* & STAG2 E1209* \\
\hline TAF1 R12330 & TAF1 R12330 \\
\hline ATRXE2246* & ATM S131* \\
\hline CTCF R49C & BCORL1E1253* \\
\hline KIT С42* & ESR1 N519S \\
\hline \multirow[t]{2}{*}{ SPTA1 R1046* } & SDHA R75* \\
\hline & SPEN E1043* \\
\hline
\end{tabular}

${ }^{A}$ Mutations common to both specimens are in bold. ${ }^{\mathrm{B}} P O L E$ mutations common to both specimens. Of note, the POLE R114* mutation was reported as a VUS in both samples.

POLE $\left(\mathrm{R} 114^{*}\right)$, consistent with inactivation of the WT allele; these features are associated with an ultramutator phenotype $(15,16,20)$. The presence of 32 and 33 potentially pathogenic mutations represents an exceptionally high mutation frequency. In addition, there were a large number of single-nucleotide variants classified as variant of unknown significance (VUS): 116 in the primary sample and 159 in the recurrence, with 83 VUS shared between the tumors. Sequencing of germline DNA performed on the same platform demonstrated that only 3 of the variants identified in both tumor samples were also present in the germline DNA (see Supplemental Methods and Supplemental Table 1; supplemental material available online with this article; doi:10.1172/JCI84940DS1). All 3 germline variants had been classified as VUS in tumor sequencing. The remaining mutations identified in tumor sequencing, including POLE mutations and all variants identified as potentially pathogenic, were somatic mutations. The distribution of shared and private somatic mutations in the 2 samples is shown in Figure 1E. This number of somatic mutations found in the set of 315 genes assayed can be extrapolated to an estimated total mutational burden of approximately 4,500 and 6,500 nonsynonymous mutations in the whole exome in the primary and metastatic samples, respectively. These estimates are consistent with the high mutational burden and ultramutator phenotype reported for POLE-mutant endometrial cancers $(15,16,20)$.

To further investigate the mutational burden in endometrial cancers, data from a set of 252 deidentified endometrial cancers sequenced using the FoundationOne assay were analyzed. Twenty-three (9.1\%) of these mostly advanced/metastatic endometrioid endometrial cancers had sequence variants in POLE. In this subset, the mean numbers of variants identified as likely pathogenic and VUS were $21.2 \pm 4.1$ and $82.2 \pm 25$, respectively, compared with mean values of $7.5 \pm 0.5$ likely pathogenic variants and $12.8 \pm 2.6$ VUS in $P O L E$-WT cases $(P<0.005$ and $P=0.015$, respectively). Thus, similarly to The Cancer Genome Atlas (TCGA) findings in mostly primary endometrial cancers, a clear subset of advanced/recurrent endometrial cancers also harbor POLE mutations and are associated with a high mutational burden.

Endometrial cancers harboring POLE mutations, approximately $10 \%$ of endometrial cancers analyzed in TCGA (20), have a much higher nonsynonymous mutational burden than other classes of endometrial cancers, including those with a microsatellite instability (MSI) phenotype (Figure 2A). To determine whether POLE-mutant cancers were associated with an immune signature, we analyzed RNA-sequencing data from endometrioid endometrial cancer that was previously deposited in TCGA (20). Serous endometrial cancers were not included, as they appear to be a distinct class of cancers with different patterns of mutations and copy number alterations (20). Endometrioid endometrial cancers were divided into 3 categories: (a) POLE-mutant cancer, (b) POLE-WT tumors with MSI phenotype, and (c) POLE-WT tumors with microsatellite stable (MSS) phenotype. A heat map of differentially expressed immunerelated genes (Figure 2B) demonstrated that POLE-mutant endometrioid endometrial cancers have high expression of a large set of immune-related genes compared with MSS cancers. The MSI tumors appear to have an intermediate phenotype, having lower expression of immune signature genes than POLEmutant tumors, but higher than MSS tumors.

In the TCGA data set, POLE-mutant cancers have higher expression of several genes encoding for immune checkpoint-related proteins, including PD-L1 and PD-L2, than either MSI or MSS endometrioid cancers (Figure 3A). Similarly, POLE-mutant cancers also showed higher expression of $\mathrm{T}$ cell markers such as CD8A, PD-1, and cytotoxic T lymphocyte-associated protein-4 (CTLA-4) (Figure 3A), suggesting the presence of a preexisting T cell infiltrate. An estimate of leukocyte subsets that may be contributing to the immune gene expression signature was generated using CIBERSORT (https://cibersort.stanford.edu/) (22). As shown in Figure 3B, POLE mutant endometrial cancers had significantly higher estimated proportions of $\mathrm{CD} 8^{+} \mathrm{T}$ cells, $\mathrm{T}$ follicular helper cells, M1 macrophages, and activated NK cells than MSS cancers, with MSI cancers having intermediate levels. There was no difference in the 
A

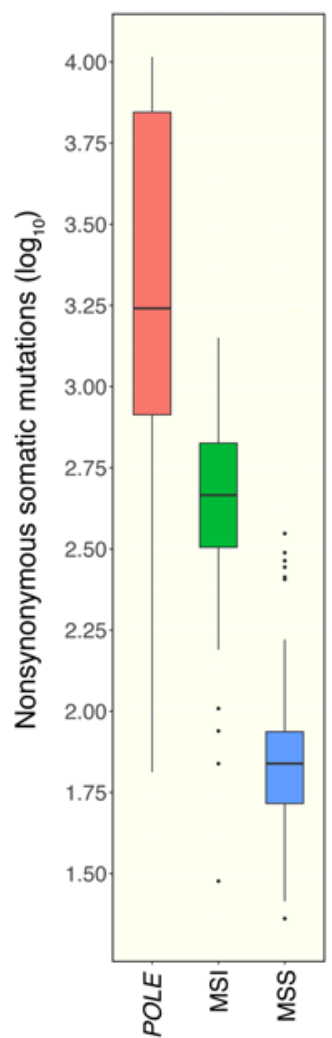

Figure 2. Comparison of immune markers in POLE, MSI, and MSS endometrial cancers in the TCGA data set. (A) The relative numbers of nonsynonymous mutations found in POLE-mutant, MSI, and MSS endometrial cancers. (B) Heat map showing relative expression of differentially expressed immune-related genes in POLE-mutant, MSI, and MSS endometrial cancers. Number of samples: 27 POLE, 64 MSI, 104 MSS. Box plots (shown in A) use the following default convention: the horizontal line represents the median value, the box covers the interquartile range (IQR), the whiskers cover values within 1.5 IQR beyond the box, and values beyond 1.5 IQR are represented as dots.
B

POLE

MSS

MSI

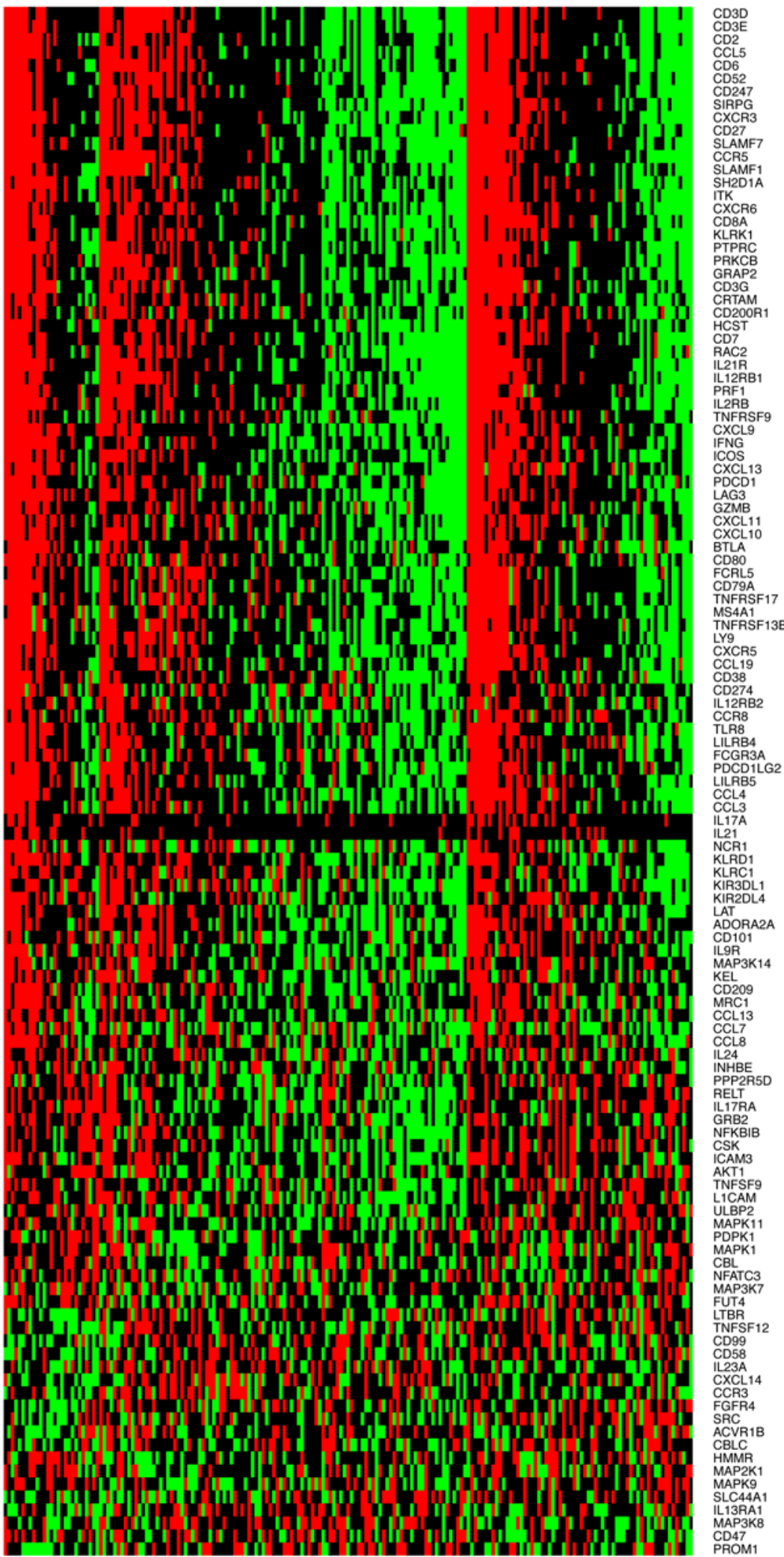




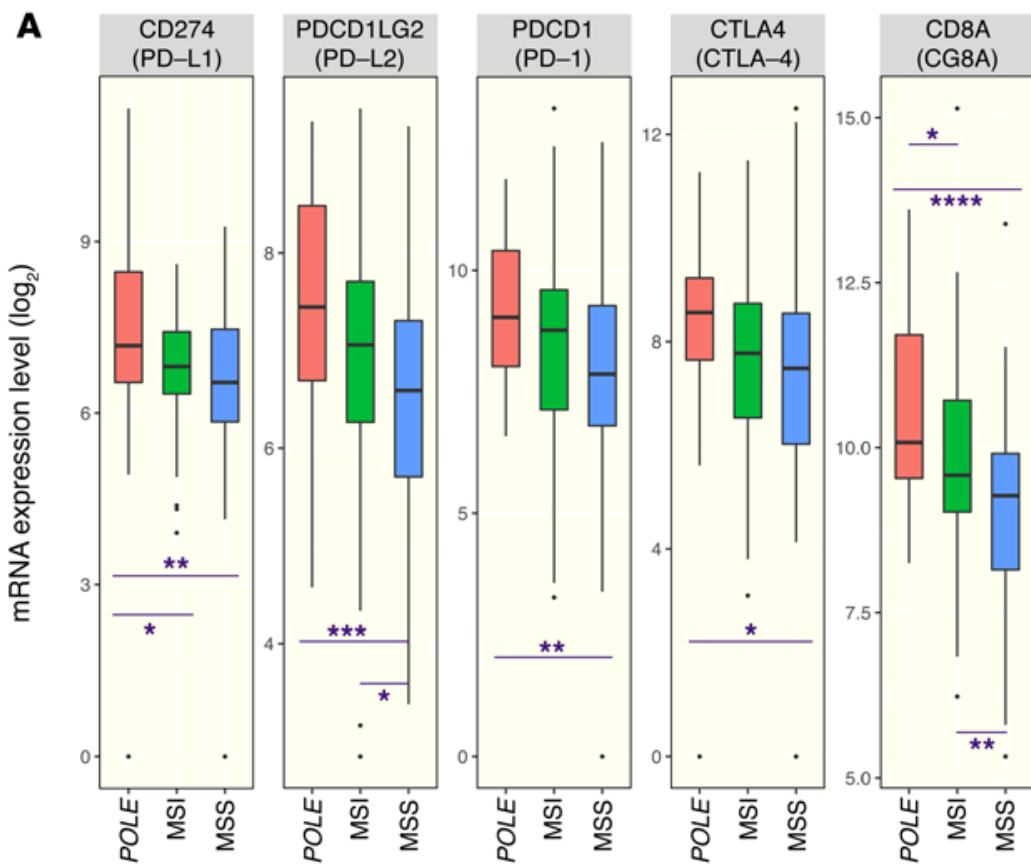

C
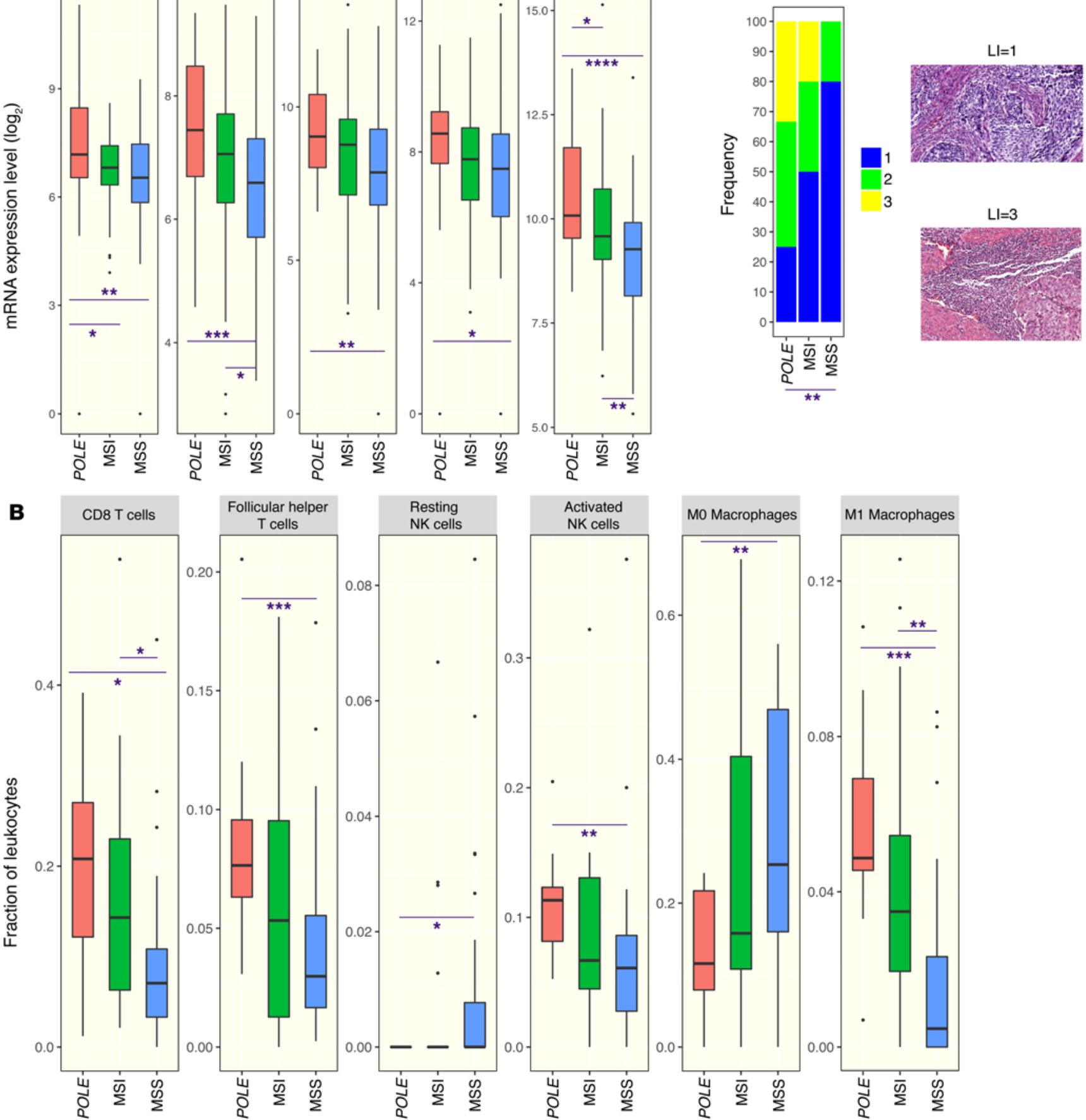

Figure 3. Expression of immune signatures in MSS, MSI, and POLE endometrial cancers in the TCCA endometrial cancer data set. (A) Expression of a set of immune checkpoint and lymphocyte-associated genes as assayed by RNA sequencing in POLE-mutant, MSI, and MSS endometrial cancers. (B) Estimated proportion representation of some leukocyte subsets, as calculated by CIBERSORT, for POLE-mutant, MSI, and MSS endometrial cancers. (C) Distribution of lymphocyte infiltration scores in histological images of a set of POLE-mutant, MSI, and MSS endometrial cancers. Representative images associated with scores of 1 and 3 are shown to the right. Original magnification, $\times 20$. A 2-sided Wilcoxon rank-sum test was used in all cases to determine $P$ values. $P<0.05$ was considered statistically significant. Number of samples: 27 POLE, 64 MSI, 104 MSS (A); 12 POLE, 20 MSI, 28 MSS (B); 12 POLE, 10 MSI, 10 MSS (C). For the remaining samples not shown in B, CIBERSORT provided a $P$ value $\geq 0.05$. Box plots (shown in $\mathbf{B}$ and $\mathbf{C}$ ) use the following default convention: the horizontal line represents the median value, the box covers the interquartile range (IQR), the whiskers cover values within 1.5 IQR beyond the box, and values beyond 1.5 IQR are represented as dots. ${ }^{*} P<0.05 ;{ }^{* *} P<0.01 ;{ }^{* * *} P<0.001 ;{ }^{* * *} P<0.0001$. 
estimated proportion of resting or activated dendritic cells or M2 macrophages between these groups (data not shown).

To determine whether POLE-mutant status was associated with histologic presence of lymphocytic infiltration, high-resolution images of H\&E-stained sections of individual endometrial cancers from TCGA were analyzed. Endometrial cancers that had POLE mutations, MSI phenotype, and MSS phenotype were analyzed in a blinded fashion and scored on a scale of 1 to 3 for the presence of lymphocytic infiltration. POLE-mutant endometrial cancers had a significantly higher extent of lymphocytic infiltration than MSS tumors; MSI tumors again had an intermediate phenotype, with a trend for more infiltration than MSS tumors but less than POLE-mutant tumors, although this did not reach statistical significance (Figure $3 \mathrm{C}$ ).

Both POLE and DNA polymerase delta 1 (POLD1) mutations have been noted in patients with non-small cell lung cancer who are responsive to pembrolizumab and are associated with high mutational burdens (11). Furthermore, mismatch repair status has been shown to predict clinical benefit from pembrolizumab in a clinical trial of both colorectal and noncolorectal cancers (13). The mutational burden seen in POLE-mutant endometrial cancers is an order of magnitude greater than that seen in endometrial cancers with the MSI phenotype (20). Gene-expression data from the TCGA (Figures 2 and 3) and several recent studies also show that $P O L E$-mutant cancers have higher expression of immune checkpoint proteins and $\mathrm{T}$ cell markers than MSI endometrioid endometrial cancers (17-19), suggesting that these tumors may be even more responsive to immunotherapy. Of note, the majority of POLE-mutant tumors have an MSS phenotype (23) and would not be identified by MSI assays. We propose further clinical investigation with immunotherapy specifically targeting endometrial and other cancers with $P O L E$ and POLD1 mutations, with translational clinical trials currently in development.

\section{Methods}

Tumor sequencing. Formalin-fixed tumor samples and DNA extracted from peripheral blood specimens were analyzed by a comprehensive genomic profiling assay performed on indexed, adaptorligated, hybridization captured libraries targeting all exons of 315 cancer-related genes at a commercial CLIA-certified laboratory, Foundation Medicine.

Data collection and analysis. Details regarding the source and analysis of mRNA expression data and analysis of histological images are summarized in Supplemental Methods.
Statistics. A 2-sided Wilcoxon rank-sum test was used in all cases to determine $P$ values. A $P$ value of less than 0.05 was considered statistically significant.

Study approval. The patient described in this report was enrolled in a clinical trial conducted at CINJ. The trial was approved by an institutional review board at CINJ, and the patient provided informed consent prior to study enrollment.

\section{Author contributions}

JMM drafted the manuscript, identified and treated patient, designed experiments, and interpreted data. AP designed experiments and performed computational analyses. HZ performed analyses of pathological specimens. KH provided direction on scope of analyses, interpreted data, and edited the manuscript. SD was involved in patient care. KL was involved in patient care. LS performed analyses of radiologic evidence. MNS provided direction on scope of analyses and was involved in patient care. LRR provided direction on scope of analyses and edited the manuscript. HLK provided direction on scope of analyses and edited the manuscript. SA acquired data and performed data analyses. JSR acquired data and performed data analyses. DCP performed data analyses. GB designed experiments and performed computational analyses. EPW provided direction on the scope of analyses. RSD provided direction on the scope of analyses and edited the manuscript. AL provided direction on the scope of analyses and edited the manuscript. JC provided direction on the scope of analyses and edited the manuscript. SG drafted the manuscript, designed experiments, and interpreted data. All authors have reviewed and approved the content in this manuscript.

\section{Acknowledgments}

This research was supported by the Functional Genomics and Biospecimen Repository Shared Resource(s) of the Rutgers CINJ (P30CA072720) and a generous gift to the Genetics Diagnostics to Cancer Treatment Research Initiative of the Rutgers CINJ and RUCDR Infinite Biologics. Funding for this research was also provided by Merck and Co.

Address correspondence to: Shridar Ganesan or Janice M. Mehnert, Rutgers Cancer Institute of New Jersey, 195 Little Albany Street, New Brunswick, New Jersey 08903, USA. Phone: 732.235.5211; E-mail: ganesash@cinj.rutgers.edu (S. Ganesan). Phone: 732.235.8945; E-mail: mehnerja@cinj.rutgers.edu (J.M. Mehnert). Hua Zhong's present address is: Department of Pathology, Community Medical Center Barnabas Health, Toms River, New Jersey, USA.
1. Robert C, et al. Nivolumab in previously untreated melanoma without BRAF mutation. $N$ Engl J Med. 2015;372(4):320-330.

2. McDermott DF, et al. Survival, durable response, and long-term safety in patients with previously treated advanced renal cell carcinoma receiving nivolumab. J Clin Oncol. 2015;33(18):2013-2030.

3. Gettinger SN, et al. Overall survival and longterm safety of nivolumab (anti-programmed death 1 antibody, BMS-936558, ONO-4538) in patients with previously treated advanced non-small-cell lung cancer. J Clin Oncol. 2015;33(18):2004-2012.
4. Rizvi NA, et al. Activity and safety of nivolumab, an anti-PD-1 immune checkpoint inhibitor, for patients with advanced, refractory squamous non-small-cell lung cancer (CheckMate 063): a phase 2, single-arm trial. Lancet Oncol. 2015;16(3):257-265.

5. Ansell SM, et al. PD-1 blockade with nivolumab in relapsed or refractory Hodgkin's lymphoma. N Engl J Med. 2015;372(4):311-319.

6. Ott PA, et al. Pembrolizumab (MK-3475) in patients (pts) with extensive-stage small cell lung cancer (SCLC): Preliminary safety and efficacy results from KEYNOTE- 028. JClin Oncol. 2015;33(suppl):7502.

7. Varga A, et al. Antitumor activity and safety of pembrolizumab in patients (pts) with PD-L1 positive advanced ovarian cancer: Interim results from a phase Ib study. JClin Oncol. 2015;33(suppl):5510.

8. Larkin J, et al. Combined nivolumab and ipilimumab or monotherapy in untreated melanoma. NEngl JMed. 2015;373(1):23-34.

9. Saltz LB. Perspectives on value. Presented at: The Plenary Session of the 2015 American Society of Clinical Oncology Annual Meeting; May 31, 2015; Chicago, Illinois, USA. 
10. Topalian SL, et al. Survival, durable tumor remission, and long-term safety in patients with advanced melanoma receiving nivolumab. JClin Oncol. 2014;32(10):1020-1030.

11. Rizvi NA, et al. Cancer immunology. Mutational landscape determines sensitivity to PD-1 blockade in non-small cell lung cancer. Science. 2015;348(6230):124-128.

12. Snyder A, et al. Genetic basis for clinical response to CTLA- 4 blockade in melanoma. $N$ Engl J Med. 2014;371(23):2189-2199.

13. Le DT, et al. PD-1 blockade in tumors with mismatch-repair deficiency. $N$ Engl JMed. 2015;372(26):2509-2520.

14. Van Allen EM, et al. Genomic correlates of response to CTLA- 4 blockade in metastatic melanoma. Science. 2015;350(6257):207-211.
15. Heitzer E, Tomlinson I. Replicative DNA polymerase mutations in cancer. Curr Opin Genet Dev. 2014;24:107-113.

16. Hussein YR, et al. Clinicopathological analysis of endometrial carcinomas harboring somatic POLE exonuclease domain mutations. Mod Pathol. 2015;28(4):505-514.

17. van Gool IC, et al. POLE proofreading mutations elicit an anti-tumor immune response in endometrial cancer. Clin Cancer Res. 2015;21(14):3347-3355.

18. Bellone $\mathrm{S}$, et al. Polymerase epsilon (POLE) ultramutated tumors induce robust tumor-specific $\mathrm{CD}^{+} \mathrm{T}$ cell responses in endometrial cancer patients. Gynecol Oncol. 2015;138(1):11-17.

19. Howitt BE, et al. Association of POLE-mutated and MSI endometrial cancers with an elevated number of tumor-infiltrating and peritumoral lymphocytes and higher expression of PD-L1. JClin Oncol. 2015;33(suppl):5511.

20. Kandoth C, et al. Integrated genomic characterization of endometrial carcinoma. Nature. 2013;497(7447):67-73.

21. Garon EB, et al. Pembrolizumab for the treatment of non-small-cell lung cancer. $N$ EnglJ Med. 2015;372(21):2018-2028.

22. Newman AM, et al. Robust enumeration of cell subsets from tissue expression profiles. Nat Methods. 2015;12(5):453-457.

23. Billingsley CC, Cohn DE, Mutch DG, Stephens JA, Suarez AA, Goodfellow PJ. Polymerase epsilon (POLE) mutations in endometrial cancer: clinical outcomes and implications for Lynch syndrome testing. Cancer. 2015;121(3):386-394. 